\title{
Transparent and Shaped Stiffness Reflection for Telesurgery
}

\author{
Bert Willaert, Pauwel Goethals, Dominiek Reynaerts, \\ Hendrik Van Brussel and Emmanuel B. Vander Poorten \\ Department of Mechanical Engineering, K.U.Leuven \\ Belgium
}

\section{Introduction}

The motivation behind the work presented in this chapter is minimal access telesurgery with reliable haptic feedback. Minimal access surgery (MAS) is performed via small incisions in the body, through which long rigid instruments are inserted along with a camera. Telesurgery refers to surgical operations performed by robotic instruments, the slave, commanded by a surgeon through one or more complex robotic joysticks, the master. Nowadays, the only commercially available master-slave system for minimal access surgery is the Da Vinci Surgical System, which is frequently used for MAS (Hockstein et al., 2007). The benefits of telesurgical laparoscopy over conventional laparoscopy include increased number of degrees of freedom, elimination of tremor, 3D visualization, possible motion scaling and an ergonomic position for the surgeon (Corcione et al., 2005; Nguan et al., 2008). Moreover, the master-slave concept enables the surgeon to be outside the direct environment of the patient. In 2001 e.g., the first transatlantic surgical operation, a laparoscopic cholecystectomy, was performed on a patient in Strasbourg, France by a surgeon situated in New York, United States (Marescaux et al., 2001).

Unfortunately, current telesurgical systems do not provide haptic feedback, which means that the surgeon loses his/her sense of touch. This decreases the efficiency of the surgeon and can result in collateral tissue damage (Bethea et al., 2004; De et al., 2007; Famaey et al., 2009). Several studies have shown that haptic feedback would be able to increase the precision of telesurgery and lower the interaction forces with the tissue (Deml et al., 2005; Tholey et al., 2005; Wagner et al., 2002). However, achieving a system with haptic feedback that accurately represents the feeling of soft tissue, while maintaining stability under all circumstances, is nontrivial and remains a big challenge. A first issue, which is not discussed in this chapter, concerns the design of a robust accurate force-measurement system that integrates well in the surgical environment (Peirs et al., 2004; Seibold et al., 2005; Willaert et al., 2009a; Zemiti et al., 2006). A second issue concerns the control itself of such a master-slave system. During MAS, the environment of interest consists mainly of soft tissue although also interactions with hard contacts can occur (i.e. contact with bone or another instrument). Based on the raw data acquired by Walraevens et al. (2008) (cardiovascular tissue) and by Rosen et al. (2008) (abdominal organs) one can estimate that the maximum stiffness is in the order of $1500 \mathrm{~N} / \mathrm{m}$ when interacting with soft tissue. When contacting bone, the stiffness can be 
up to $8000 \mathrm{~N} / \mathrm{m}$ (Bankman et al., 1990). The design of haptic teleoperation controllers for interaction with these types of environments is receiving growing interest from the research community (Cavusoglu et al., 2002; De Gersem et al., 2005a; Malysz \& Siroupour, 2007; Misra \& Okamura, 2006; Tavakoli et al., 2006) and forms the topic of this chapter.

To situate the controllers studied in this chapter among the large spectrum of bilateral teleoperation controllers, we distinguish three main concepts based on the rendering of the haptic feedback. Controllers of the first concept send all sensory data directly from the master to the slave and vice versa. All implementations of the "four-channel" controller (Lawrence, 1993; Yokokohji \& Yoshikawa, 1994) are examples of such controllers. Controllers of the second concept still exchange the sensory data directly between master and slave, but the sensory data are also used to create a model of the environment and/or human operator. That model is used to adapt one or more parameters of the controller online. Preusche et al. (2002) use an estimate of the environment stiffness to adapt the stiffness of the position controller of the slave, while (Love \& Book, 2004) use this estimate to change the extra damping of the master. Controllers of the third concept provide model-based haptic feedback and no sensory data is sent explicitly to the master. The sensory data of the slave are used to create a model of the environment and that model is sent back to the master to create the haptic sensation. Depending on the application, this model can have different levels of complexity. Hashtrudi-Zaad \& Salcudean (1996) assume a mass-spring-damper model for the environment while the models of Funda \& Paul (1991) and of Mitra \& Niemeyer (2008) only describe a rigid wall at a variable location.

The main goal of this chapter is to discuss and demonstrate some major benefits of the third control concept, especially when used for telesurgery: (1) Model-based controllers are well-suited to deal with some typical problems associated with most modern surgical robots. As mentioned by Funda \& Paul (1991); Hashtrudi-Zaad \& Salcudean (1996); Ji et al. (2005); Kuan \& Young (2003); Mitra \& Niemeyer (2008); Tzafestas et al. (2008), a teleoperation system with model-based haptic feedback is more robust with respect to communication time delay. The increased robustness is even not restricted to the problem of communication time delay. This chapter shows that model-based controllers also offer also an increased robustness with respect to the restricted position tracking of the slave and the presence of low-pass filters. Note that elimination of surgical tremor by filtering the commands of the surgeon is often mentioned as one of the benefits of telesurgery (Hockstein et al., 2007; Okamura, 2004), but the use of such a filter can be conflicting with the requirement of reliable haptic feedback. To show the beneficial properties of the third concept, this chapter describes a practical implementation of a controller of this concept, which will be referred to as the Stiffness Reflecting controller (SRC). Based on experiments, the robustness of this controller over a controller of the first concept, namely the classical Direct Force Feedback controller (DFF), is demonstrated. (2) An additional benefit, next to the increased robustness, is that model-based haptic feedback offers the possibility to shape the reflected environment impedance. A generalized version of the SRC was originally proposed in order to maximize the stiffness discrimination ability of the surgeon during telesurgery (De Gersem et al., 2005b). A psychophysics experiment demonstrated that human stiffness perception for interactions with soft environments follows Weber's law (Weber, 1834), i.e. the change in stiffness that can just be discriminated is a constant fraction of the nominal stiffness, this fraction being 8-12 \% (De Gersem, 2005c). To increase the stiffness discrimination ability, this generalized version of the SRC, applies a nonlinear shaping function to the estimated stiffness before 
reflection to the master. This principle of enhanced sensitivity (De Gersem et al., 2005a;b; Malysz \& Siroupour, 2007; Son \& Lee, 2008), allows the human operator to feel a difference in stiffness that is smaller than one he/she can detect by manual palpation. This chapter describes a practical implementation of the generalized version of the SRC (gSRC) and shows the feasibility of enhanced sensitivity based on a set of psychophysical user tests.

The layout of this chapter is as follows: Section 2 describes the concept of transparency and discusses the relevance of transparency for the application of telesurgery. Section 3 describes both the Stiffness Reflecting Controller with its generalized form and the Direct Force Feedback controller. The implementation of these controllers on an experimental setup is described in Section 4. Experiments comparing the performance and the stability of these controllers are described and discussed in Section 5, while the experiments on enhanced sensitivity are described and discussed in Section 6. The chapter ends with a discussion in Section 7 and a conclusion in Section 8.

\section{Transparency for telesurgery}

In literature, several definitions and indices for transparency have been proposed. According to Handlykken \& Turner (1980) the ideal teleoperation system behaves as a massless infinitely stiff connection between the end-effector of the master and the slave. In that case, the system is completely transparent and the human operator feels as if directly manipulating the remote environment. Yokokohji \& Yoshikawa (1994) state that the ideal response is obtained when the positions and forces at the master $\left(x_{h}, F_{h}\right)$ and slave $\left(x_{e}, F_{e}\right)$ are identical:

$$
\forall t:\left\{\begin{array}{l}
x_{e}(t)=x_{h}(t) \\
f_{h}(t)=f_{e}(t)
\end{array}\right.
$$

Lawrence (1993) looks at the impedance $Z_{t h}$ "felt" by the human operator. The subscript $t h$ denotes 'transmitted to the human operator'. For an environment impedance $Z_{e}$, the transparency condition can be written as:

$$
Z_{t h}=Z_{e} \text {. }
$$

Note that impedance is defined as $\frac{F}{v}$. Elaborating on this last definition (2), this section explains the concepts of stiffness transparency and enhanced stiffness sensitivity.

\subsection{Stiffness Transparency}

The perception and differentiation of the tissue's stiffness is essential during surgical palpation tasks (De Gersem et al., 2005a). Palpation is the technique of examining parts of the body by touching and pressing them. For example when performing tasks such as quality verification of a suture, localization of arteries or veins beneath fatty or surrounding tissue (Scott \& Darzi, 1997), or identification of primary and metastatic cancer (Ota, 1995), stiffness discrimination is important. Therefore, this chapter focusses on the reflection of the stiffness properties of the environment through a bilateral teleoperation controller.

The concept of Stiffness Transparency defines how accurately the human operator feels the environment stiffness $\left(k_{e}\right)$ through the teleoperation system. An indicative measure, is the difference $\xi$ between the real environment stiffness and the stiffness felt by the human operator when manipulating the master quasi-statically. This can be expressed in the frequency domain for a known environment stiffness $k_{e}$ as:

$$
\xi=\lim _{s \rightarrow 0}\left(s . Z_{t h}\left(s, k_{e}\right)\right)-k_{e} .
$$


When experimental data are available, $\xi$ can also be expressed in the time-domain:

$$
\xi(t)=\frac{\triangle F_{h}(t)}{\triangle x_{h}(t)}-k_{e}(t)
$$

In literature, experimental results are typically shown with a position versus time and a force versus time plot, following the definition of the ideal response of Yokokohji \& Yoshikawa (1994). However, from such plots, it is difficult to analyse what the human operator feels. For this purpose, it is more useful to present the experimental data on force versus position plots, as done by De Gersem et al. (2005a); Mahvash \& Okamura (2007); Tzafestas et al. (2008); Willaert et al. (2008b). Both ways of plotting the experimental data in the time-domain are employed in this chapter. Note that the frequency domain analysis is most appropriate to see all linear dynamics felt by the human operator, while the time domain analysis can also show the effect of nonlinear phenomena present in the teleoperation system.

Inspired by the idea of Impedance Reflection (De Gersem et al., 2005b; Hannaford, 1989; Hashtrudi-Zaad \& Salcudean, 1996), Willaert et al. (2008b) presented the Stiffness Reflecting Controller (the SRC) for the purpose of stiffness transparency. This is a controller of the third concept for which haptic feedback is generated through reflection of the estimated environment stiffness to the master. The implementation of this controller will be discussed in detail in Section 3.

\subsection{Enhanced Stiffness Sensitivity}

As stated above, differentiation of tissue stiffness is important during surgical procedures. Since human perception of stiffness is limited both by absolute and differential thresholds, a perfectly stiffness-transparent system might not be sufficient for some differentiation tasks. To overcome the absolute thresholds, existing linear scaling techniques can be used, while for the differential thresholds, these techniques offer no solution. The problem of the differential thresholds is addressed in this chapter. Inspired by the idea of Impedance Shaping (Colgate, 1993), De Gersem et al. introduced the idea to overcome the differential thresholds by means of teleoperation control (De Gersem et al., 2005a;b). As stated in the introduction, the minimal change in stiffness that can be discriminated by a human operator is a constant fraction $c$ of the nominal stiffness. For soft environments this fraction $c$ was found to be $8-12 \%$ (De Gersem, 2005c). To increase the stiffness discrimination ability, a relative change $\delta k_{e}$ around the nominal environment stiffness $k_{e, n}$ should induce a higher relative change in stiffness felt by the operator:

$$
\delta k_{t h}=\frac{\Delta k_{t h}}{k_{t h, n}}>\frac{\Delta k_{e}}{k_{e, n}}=\delta k_{e} .
$$

Introducing an extra design parameter $\sigma$, and requiring that

$$
\delta k_{t h}=\sigma \delta k_{e}
$$

makes that a tissue with stiffness $k_{e}=\left(k_{e, n}+\Delta k_{e}\right)$ feels different from $k_{e, n}$ if and only if $\delta k_{e} \geq \frac{c}{\sigma}$. Requiring that

$$
k_{t h}=K k_{e}^{\sigma},
$$

yields that one can discriminate environments with a difference in stiffness $\sigma$ times smaller than the human differential threshold for stiffness discrimination. The factor $\sigma$ can be interpreted as the sensitivity factor for discrimination. $K$ serves as a scaling factor. Here it is used to keep the absolute value of $k_{t h}$ at a similar value as $k_{e}$. As stated above, this chapter 


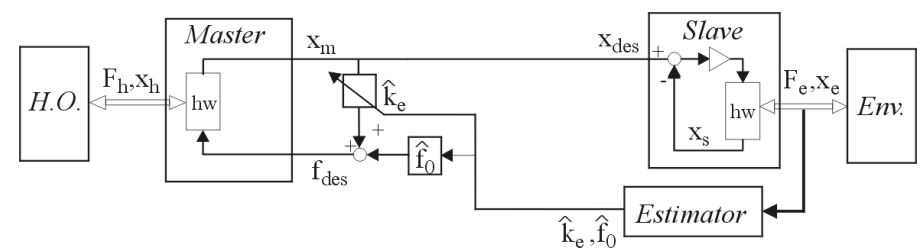

Fig. 1. The Stiffness Reflecting Controller (SRC), reflecting the estimated stiffness of the environment to an impedance controller at the master side.

only addresses the differential thresholds, although, through the parameter $K$, the presented controller can also be employed to overcome absolute thresholds. The implementation of the control law realizing expression (7) will be discussed in the following section.

\section{Controller Definition}

This section describes the definition of controllers designed for stiffness transparency and enhanced stiffness sensitivity. Experimental validation of the quality of these controllers takes place in Sections 5 and 6. The first controller is the Stiffness Reflecting controller presented by Willaert et al. (2008b). The second controller is the generalized form of the Stiffness Reflecting Controller proposed by De Gersem et al. (2005b). Both controllers will be compared to the classical Direct Force Feedback controller (DFF), described in the latter part of this section. Earlier work on soft tissue telemanipulation already described the potential of the DFF for telesurgery (Cavusoglu et al., 2002; De Gersem et al., 2005a). All controllers described are to be used with a master device of the impedance type, i.e. a system with low mass and low friction (e.g. the PHANToM). However, the implementations of the controllers can be modified in such a way that they can also be used with a master of the admittance type. For the hardware of master and slave, 1-d.o.f rigid-body models are supposed, obeying the following equations of motion:

$$
\begin{gathered}
F_{h}+\tau_{m}=M_{m} \ddot{x}_{m}+B_{m} \dot{x}_{m}, \\
\tau_{s}-F_{e}=M_{s} \ddot{x}_{s}+B_{s} \dot{x}_{s}, \\
Z_{m}=M_{m} s+B_{m}, \quad Z_{s}=M_{s} s+B_{s},
\end{gathered}
$$

with $Z_{m}$ and $Z_{s}$ representing the impedances of the master and the slave robot. Remark that for a rigid body model the positions $x_{m}$ and $x_{s}$ (the position at the motors) correspond to respectively $x_{h}$ and $x_{e}$ (the position of the end-effectors).

\subsection{The SRC scheme}

The Stiffness Reflecting Controller (SRC) originates from the idea to reflect the estimated stiffness of the environment to an impedance controller at the master side. In the SRC, depicted in Fig. 1, the slave is under position control following the master's position. While the slave follows the master, an estimator estimates the local remote environment stiffness $k_{e}$ and the offset force $f_{o}$. These parameters are related to the position of the slave $x_{e}$ and the measured interaction force $F_{e}$ by the following local, linearized force-position relationship:

$$
F_{e}=f_{o}+k_{e} \cdot x_{e}
$$

Note that the relationship $F_{e}=k_{e}\left(x_{e}-x_{0}\right)$ is not linear in the parameters to be estimated $\left(k_{e}, x_{0}\right)$. Fig. 2 shows the relation between the different parameters on a force-position curve. 
The estimates $\hat{k}_{e}$ and $\hat{f}_{o}$ are used to determine $f_{\text {des }}$, the force input for the master:

$$
f_{\text {des }}=\hat{f}_{o}+\hat{k}_{e} \cdot x_{m}+c \cdot \hat{k}_{e} \cdot \dot{x}_{m} .
$$

The last term in expression (12) is a stiffness dependent damping term (gain: $c . \hat{k}_{e}$ ), which has a significant positive effect on the stability as discussed in Section 4.1. As the considered master is of the impedance type, the force $f_{\text {des }}$ is applied in open loop to the master. To summarize, the control inputs for the master and the slave become:

$$
\begin{gathered}
\tau_{m}=-f_{\text {des }}, \\
\tau_{s}=K_{p}\left(x_{m}-x_{s}\right)-K_{v} \dot{x}_{s} .
\end{gathered}
$$

Based on this control law, the impedance "felt" by the human operator can be approximated by:

$$
Z_{t h} \approx M_{m} s+\left(B_{m}+c \cdot \hat{k}_{e}\right)+\frac{\hat{k}_{e}}{s} .
$$

As a consequence, the difference between the stiffness the human operator feels when manipulating the master quasi-statically and the real environment stiffness is:

$$
\xi=\lim _{s \rightarrow 0}\left(s \cdot Z_{t h, k_{e}}(s)\right)-k_{e}=\hat{k}_{e}-k_{e} .
$$

Depending on the correctness of the estimate, this difference approaches zero and thus the human operator feels approximately the correct environment stiffness.

The stiffness estimator used in this work is an Extended Kalman Filter. This is a well-known and widely-used recursive algorithm to estimate time-varying parameters, taking into account uncertain system dynamics and uncertainty caused by measurement noise (Kalman, 1960). For a compact tutorial on the Kalman Filter, see De Schutter et al. (1999). At each timestep, a new estimate and an associated uncertainty are calculated, given the previous estimate with its associated uncertainty and given the latest measurements. Within the Kalman filter formalism, the system's process and measurements equations are described as follows:

$$
\begin{gathered}
\mathbf{y}_{i}=A \cdot \mathbf{y}_{i-1}+B \cdot \mathbf{u}_{i-1}+\rho_{p}, \\
\mathbf{z}_{i}=H_{i} \cdot \mathbf{y}_{i}+\rho_{m},
\end{gathered}
$$

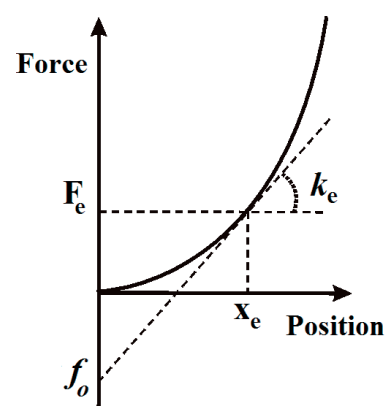

Fig. 2. The relation between the local stiffness $k_{e}$, the offset force $f_{o}$, the position $x_{e}$ and the force $F_{e}$. 
with $\mathbf{y}_{i}$ the state vector, $\mathbf{u}_{i}$ the control input and $\mathbf{z}_{i}$ the measurement vector at time step i. $\rho_{p}$ is the process model uncertainty or process noise and $\rho_{m}$ is the measurement model uncertainty. Applied to the estimation of the environment stiffness, the unknown parameters $k_{e}$ and $f_{o}$ form the state variables $\mathbf{y}_{i}=\left[f_{0}, k_{e}\right]^{T}$. Based upon the idea that the stiffness varies only slowly during surgical manipulation, the process is modelled as a random walk process with process noise $\rho_{p}$ and no control input $\mathbf{u}_{i}$. So, equation (17) reduces to:

$$
\mathbf{y}_{i}=\mathbf{y}_{i-1}+\rho_{p}
$$

$\rho_{p}$ represents Gaussian process uncertainty with zero mean and covariance matrix $Q$. Large values for the covariance matrix $Q$ result in faster convergence (e.g. when going from noncontact to contact state), but have the drawback that the estimates become more volatile. The approach to determine sensible values for the covariance matrix of the process noise is discussed in more detail in Section 4.1. The measured position $x_{e, i}$ and the interaction force $F_{e, i}$ do not allow direct estimation of $f_{0}$ and $k_{e}$ as these two unknowns are related only by the single equation (11) to $x_{e}$ and $F_{e}$. In order to decouple both estimates, the measurement equation at each time step is constructed as follows, based upon the measured position $\left(x_{e, i}\right)$ and force $\left(F_{e, i}\right)$ and the with $j$ time steps $T_{S}$ delayed position measurement $\left(x_{e, i-j}\right)$ and force measurement $\left(F_{e, i-j}\right)$ :

$$
\begin{gathered}
F_{e, i}=f_{o}+k_{e} \cdot x_{e, i} \\
k_{e}=\frac{\Delta f}{\Delta x}=\frac{F_{e, i}-F_{e, i-j}}{x_{e, i}-x_{e, i-j}} .
\end{gathered}
$$

To obtain the estimates $\hat{k}_{e}$ and $\hat{f}_{o}$, the explicit measurement equation of (18) should be reorganized into the following implicit measurement equation:

$$
h\left(\mathbf{y}_{i}, \mathbf{z}_{i}\right)=\left[\begin{array}{c}
\left.F_{e, i}-\mathbf{y}_{i}, \mathbf{z}_{i}\right)+\rho_{m}=0 \quad \text { with } \\
F_{e, i-j}-k_{e}\left(x_{e, i}-x_{e, i-j}\right) \\
F_{e, i}-f_{o}-k_{e} \cdot x_{e, i}
\end{array}\right] .
$$

Since the measurement equation is nonlinear, an Extended Kalman Filter is used. The resulting estimates $\hat{k}_{e}$ and $\hat{f}_{o}$ form the environment model (12) that is used to create the haptic feedback at the master.

\subsection{The gSRC scheme}

The gSRC scheme is a generalized version of the SRC scheme. The control inputs for the master and the slave are the same as in (13) and (14), with a generalized $f_{\text {des }}$ :

$$
f_{\text {des }}=f_{t h, o}+k_{t h} \cdot x_{m}+c \cdot \hat{k}_{e} \cdot \dot{x}_{m} .
$$

The parameters $f_{t h, o}$ and $k_{t h}$ are now a function of the estimated parameters $\hat{k}_{e}$ and $\hat{f}_{0}$, rather than being the estimates themself. To realize enhanced stiffness sensitivity following (7), $k_{t h}$ is calculated as $K \hat{k}_{e}^{\sigma}$. The parameter $f_{t h, o}$ can be obtained using the requirement that any zero interaction force $\left(F_{e}=0\right)$ at the slave side should give a zero transmitted force $\left(f_{\text {des }}=0\right)$. Using (24) and supposing quasi-static manipulation, the requirement $f_{\text {des }}=0$ can be written as:

$$
f_{t h, o}=-K \hat{k}_{e}^{\sigma} x_{m}
$$


The position tracking behaviour of the slave can be described using linear techniques. If the hardware of the slave is described by its impedance $Z_{S}$ and the local position controller by $C_{S}$, the relation between $x_{m}$ and $x_{e}$ can be written as:

$$
\begin{aligned}
X_{e} & =h_{1} X_{m}-h_{2} F_{e}, \\
\text { with } \quad h_{1} & =\frac{C_{s}}{Z_{s}+C_{s}} \\
h_{2} & =\frac{1}{Z_{s}+C_{s}} .
\end{aligned}
$$

For low-frequency manipulation, $h_{1}$ can be considered as 1 and $h_{2}$ as constant. The position tracking in time domain can now be written as:

$$
x_{e}=x_{m}-h_{2} F_{e} .
$$

For the considered case that the interaction force is zero $\left(F_{e}=0\right)$, above expressions simplify to their first term. Combining the equations (11), (25) and (27) results in:

$$
\begin{aligned}
f_{t h, o} & =-K \hat{k}_{e}^{\sigma} x_{e} \\
& =-K \hat{k}_{e}^{\sigma-1}\left(\hat{k}_{e} x_{e}\right) \\
& =K \hat{k}_{e}^{\sigma-1} \hat{f}_{o} .
\end{aligned}
$$

Fitting the last expression (28) into (24) results in:

$$
f_{\text {des }}=K \hat{k}_{e}^{\sigma-1} \hat{f}_{o}+K \hat{k}_{e}^{\sigma} x_{m}+c \hat{k}_{e} \dot{x}_{m} .
$$

Note that if $h_{2}$ is small, the slave tracking is robust with respect to external forces. In that case, using expression (28) is still acceptable for reasonably small $F_{e}$.

The parameters $f_{t h, o}$ and $k_{t h}$, being function of the estimated parameters $\hat{k}_{e}$ and $\hat{f}_{o}$, form the model that is used to create the haptic feedback at the master, following (24).

\subsection{The DFF scheme}

The Direct Force Feedback controller (DFF) is a combination of a position controller at the slave side and a force controller at the master side. The input for the slave's position controller is the measured position of the master and the input for the master's force controller is the measured interaction force at the slave side $F_{l}$. Compared to the position-controller of the SRC scheme a velocity-feedforward term is added to the position controller of the slave, as this implementation of the DFF has better stability properties (Willaert et al., 2009b). The control inputs for the motors of the master and the slave become:

$$
\begin{gathered}
\tau_{m}=-F_{e}, \\
\tau_{s}=\left(K_{v} s+K_{p}\right)\left(x_{m}-x_{s}\right) .
\end{gathered}
$$

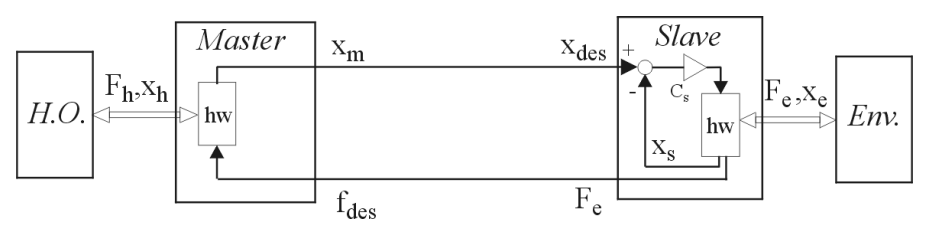

Fig. 3. The Direct Force Feedback Controller. 


\begin{tabular}{|ll|ll|}
\hline \multicolumn{2}{|l|}{ Model } & \multicolumn{2}{l|}{ Controller } \\
\hline \hline$M_{m}:$ & $0.64 \mathrm{~kg}$ & $K_{v}:$ & $80 \mathrm{Ns} / \mathrm{m}$ \\
$B_{m}:$ & $3.4 \mathrm{Ns} / \mathrm{m}$ & $K_{p}:$ & $4000 \mathrm{~N} / \mathrm{m}$ \\
$M_{s}:$ & $0.61 \mathrm{~kg}$ & & \\
$B_{s}:$ & $11 \mathrm{Ns} / \mathrm{m}$ & & \\
\hline
\end{tabular}

Table 1. Parameters of the teleoperation system

Based on this control law, the impedance felt by the human operator can be calculated:

$$
Z_{t h}=\frac{\left(M_{m} s^{2}+B_{m} s\right)\left(M_{s} s^{2}+\left(B_{s}+K_{v}\right) s+\left(K_{p}+k_{e}\right)\right)+k_{e}\left(K_{v} s+K_{p}\right)}{s\left(M_{s} s^{2}+\left(B_{s}+K_{v}\right) s+\left(K_{p}+k_{e}\right)\right)} .
$$

As a consequence, the difference between the stiffness the human operator feels when manipulating the master quasi-statically and the real environment stiffness is:

$$
\xi=\lim _{s \rightarrow 0}\left(s . Z_{t h}\left(s, k_{e}\right)\right)-k_{e}=\left(\frac{K_{p} k_{e}}{K_{p}+k_{e}}\right)-k_{e} .
$$

Therefore, the human operator feels the series connection of the real environment stiffness and the stiffness of the position controller, i.e. a stiffness smaller than the actual environment stiffness.

\section{Controller Implementation}

This section describes the implementation on a 1-d.o.f experimental master-slave setup of the controllers defined above. The experimental setup, shown in figure 4 consists of two currentdriven voice coil motors recycled from hard disk drives. On both devices, one-dimensional force sensors are mounted, measuring the interaction forces between slave and environment and between the human operator and the master (noise level: $\pm 0.05 \mathrm{~N}$ ). Linear encoders offer accurate position measurements (resolution: $1 \mu \mathrm{m}$ ). A rigid-body model for the master and the slave is chosen as the structural resonance frequencies are above $100 \mathrm{~Hz}$. The controllers are implemented on a dSPACE board, in a real time loop with a frequency of $1 \mathrm{kHz}\left(T_{\mathrm{s}}=1 \mathrm{~ms}\right)$. Table 1 summarizes the parameters for the hardware, based on a linear model identification of the setup, and the parameters for the DFF controller, employed during the experiments. The implementations of the SRC and the gSRC are described in more detail in two following sections.

\subsection{The SRC scheme}

This section describes the practical implementation of the controller defined in Section 3.1. Firstly, the position controller, see eq. (14), is tuned following standard techniques in order to obtain a good and stable step response. The resulting parameters can be found in Table 1 .

Next, the parameters of the Extended Kalman filter, i.e. the estimator, are tuned. The behaviour of this filter depends on the process noise $\rho_{p}$, the measurement model uncertainty $\rho_{m}$ and the delay ( expressed as a number of time samples: $j \cdot T_{s}$ ) between the used measurements. The covariance matrix for the measurement model uncertainty $\rho_{m}$ is fixed a priori based on 


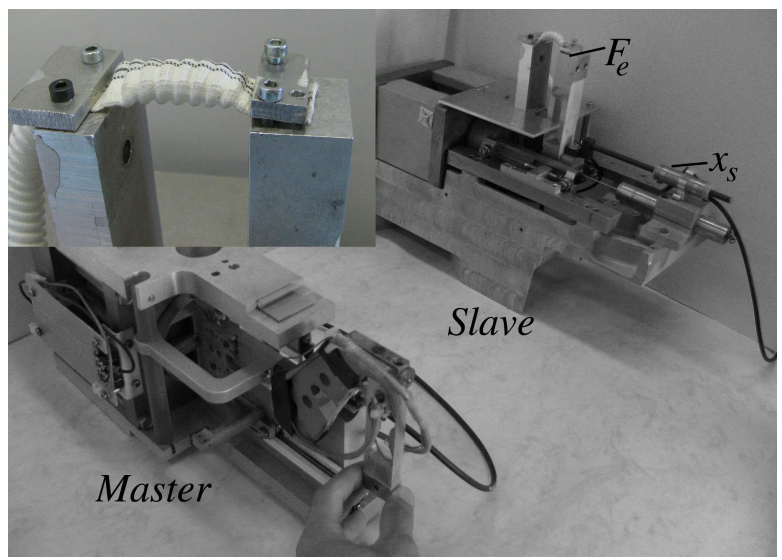

Fig. 4. The experimental 1 d.o.f. master-slave system. In detail a Dacron cardiovascular prosthesis at the slave side.

the sensor specifications:

$$
R=\left[\begin{array}{cccc}
(0.002 \mathrm{~mm})^{2} & 0 & 0 & 0 \\
0 & (0.002 \mathrm{~mm})^{2} & 0 & 0 \\
0 & 0 & (0.05 \mathrm{~N})^{2} & 0 \\
0 & 0 & 0 & (0.05 \mathrm{~N})^{2}
\end{array}\right]
$$

The process noise is a vector with zero mean and covariance matrix $Q$ :

$$
Q=\left[\begin{array}{c}
\left(q_{1} N\right)^{2} \\
\left(q_{2} N / m\right)^{2}
\end{array}\right]
$$

A number of simulation runs and experiments were performed to determine sensible values for $q_{1}, q_{2}$ and $j$. Figures 5(a) and 5(b) show the simulation data $(x, F)$ used as input to tune the estimator. White-noise is added to the force measurement signal $( \pm 0.02 \mathrm{~N})$. Figure 5(c) shows the estimates $\hat{k}_{e}$ and $\hat{f}_{o}$ for $j=12$ and different values of $q 1=q 2=q$. Figure $5(\mathrm{~d})$ shows these estimates for $q_{1}=q_{1}=0.03$ and different values of $j$. From these figures, one can see that:

- Larger values $q_{i}$ of the covariance matrix of the process noise $\rho_{p}$ result in a faster (and more correct) response to a change in environment stiffness. This is obvious as the process is defined as a random walk process in eq. (19). However, larger values of $q_{i}$ also mean that the estimator is more reactive to measurement noise. This results in more volatile estimates, which might be transferred to the human operator and disturb his/her perception of the remote environment. Therefore, tuning the covariance matrix of the proces noise boils down to finding a compromise between having sufficiently smooth transients and sufficiently fast convergence to correct estimates $\hat{k}_{e}$ and $\hat{f}_{o}$. Note that this compromise depends strongly on the signal-to-noise ratio of the position and force measurements at the slave. The better the signal-to-noise ratio of the measurements, the larger the values $q_{i}$ that can be chosen. 


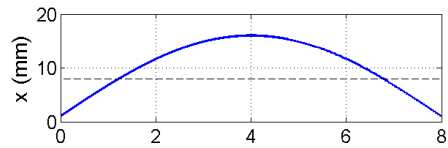

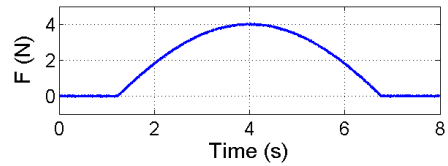

(a)

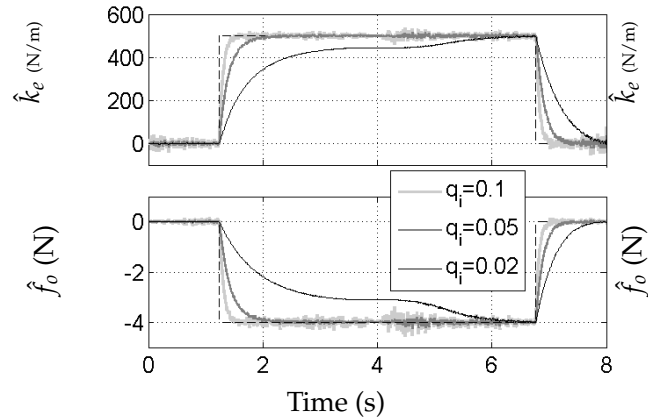

(c)

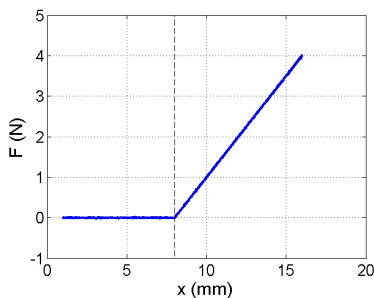

(b)
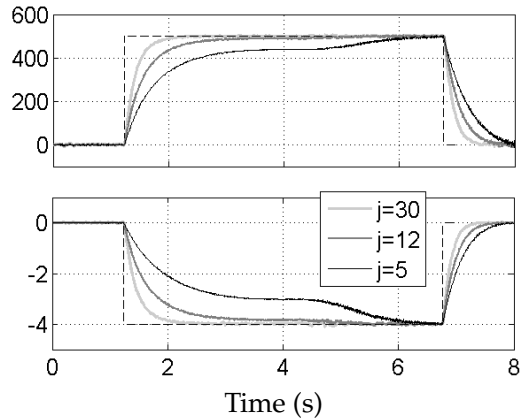

(d)

Fig. 5. The motion and force profiles (a), simulating an interaction with a perfect spring, with stiffness $k_{e}=500 \mathrm{~N} / \mathrm{m}(\mathrm{b})$, used to analyse the behaviour of the estimator. The estimates $\hat{k}_{e}$ and $\hat{f}_{o}$ are displayed for this simulation data for (c) $j=12$ and different values of $q_{1}=q_{2}=q_{i}$ and (d) for $q_{1}=q_{2}=0.03$ and different values of $j$. The theoretically correct value is indicated as a dashed line in (c) and (d).

- A larger time shift $j \cdot T_{s}$ between the two data sets $\left(x_{e, i}, F_{e, i}\right)$ and $\left(x_{e, i-j}, F_{e, i-j}\right)$, also results in a faster (and more correct) response to a change in environment stiffness. This can be explained as follows: the update equation of the form $\hat{y}_{i}=\tilde{y}_{i}+K_{k}\left(c-h\left(\tilde{y}_{i}, z_{i}\right)\right)$ contains an error term $\left(c-h\left(\tilde{y}_{i}, z_{i}\right)\right)$ described by (23). For a particular velocity the absolute values of both $\left(F_{e, i}-F_{e, i-j}\right)$ and $\left(x_{e, i}-x_{e, i-j}\right)$ are larger for a longer delay $j \cdot T_{S}$ in contact mode. Thus, the first error term in (23) increases as the delay increases, which results in a faster response. However, also here a compromise is at hand, as the very initial response to a change in environment stiffness is slower for a larger value $j$. Note that this is only problematic for very abrupt changes in environment stiffness. The initial contact with a perfectly linear spring shows such an abrupt change. When contacting soft tissue in a surgical scenario, the initial contact is typiccaly not problematic, due to the low stiffness of soft tissue at small strain. On the other hand, the change in environment stiffness at the moment of motion reversal could be problematic. Both cases are shown in figure 6.

Based on these findings and trials on the experimental setup, the covariance matrix has been set to:

$$
Q=\left[\begin{array}{c}
(0.1 N)^{2} \\
(0.1 \mathrm{~N} / \mathrm{m})^{2}
\end{array}\right]
$$

and the time shift between the two data sets is set to $\left(12 \cdot T_{S}\right)$. Ideally, the estimator uses position measurements and force measurements acquired at the end-effector of the slave. Here, the force measurement is actually done at the end-effector $\left(F_{e}\right)$ and the position measurement 
(a)

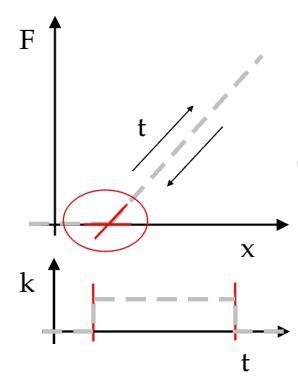

(b)

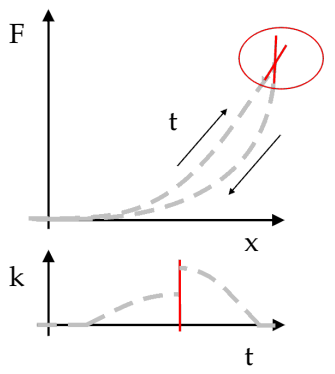

Fig. 6. Abrupt changes in stiffness (a) at the initial contact with a linear spring and (b) at the reversal of motion when manipulating soft tissue.

is done at the motor of the slave $\left(x_{s}\right.$ instead of $\left.x_{e}\right)$. For the 1 d.o.f. master-slave setup used here, this is only a theoretical difference as both the slave and the master behave as a rigidbody for frequencies below $100 \mathrm{~Hz}\left(x_{s} \approx x_{e}\right)$.

Note that in order to have a smooth feeling in free motion $\left(F_{e} \approx 0\right)$ and to avoid problems with transition from free motion to contact, $f_{d e s}$ is set to zero as long as the measured interaction force $F_{e}$ is smaller than $0.2 \mathrm{~N}$.

The last aspect of the implementation of the SRC, discussed in this section, is its stability. The analysis of the stability of this controller is not straightforward. Due to the presence of the Extended Kalman Filter, classical tools such as closed-loop stability and frequency-domain passivity cannot be used. De Gersem et al. (2005b) suggest that the SRC decouples the master and the slave. In practice, however, this is only partially true due to the existence of estimation errors and estimation lag. Especially when contacting hard objects, i.e. for a sudden change in environment stiffness, stability can be problematic with the SRC. For an intuitive understanding of the stability properties of the SRC, one can observe that the stability properties of this controller shift from those of a haptic controller for interaction with a virtual wall to those of a Direct Force Feedback teleoperation controller (DDF), depending on the ratio $\frac{q_{1}}{q_{2}}$. Figure 7 shows the effect of varying $q_{2}$ for a fixed $q_{1}$. From this figure, it is clear that for decreasing
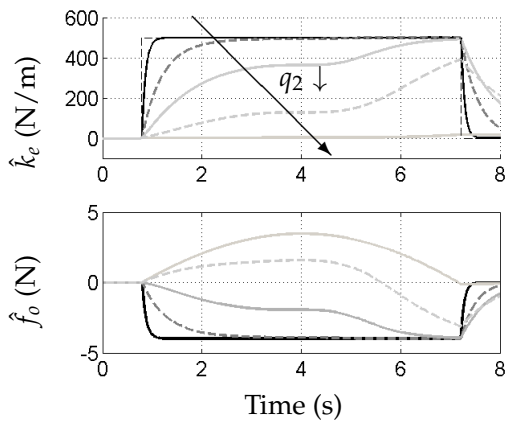

Fig. 7. The estimates $\hat{k}_{e}$ and $\hat{f}_{o}$ for the simulation data in figure 5(a) for decreasing values of $q_{2}$ (0.1-0.020.005-0.003-0.001) while $q_{1}$ is kept constant $\left(q_{1}=0.1\right)$. 
values of $q_{2}$, the error on the estimate of the environment stiffness increases. Stated differently, the estimate $\hat{k}_{e}$ tends more and more to zero than to the correct environment stiffness, while the estimate $\hat{f}_{o}$ tends towards $F_{e}$. For the limit case $\hat{k}_{e} \approx 0$ and $\hat{f}_{o} \approx F_{e}$, the force input to the master does no longer depend on the position of the master $x_{m}$ and the SRC behaves exactly as the DFF. This shows that the decoupling of the master and the slave is not absolute but depends on the properties of the estimator. The gain in robustness, mentioned in the introduction, proper to controllers of the third concept, depends on how well master and slave are decoupled. In order to maximize the decoupling, both $q_{1}$ and $q_{2}$ have to be large.

An extra measure to improve the overall stability of the system, while only minimally compromising the transparency, is the addition of a damping at the master proportional to the estimate of the environment stiffness: $c . \hat{k}_{e} \cdot \dot{x}_{m}$ (see (12)). This extra damping term has a significant positive effect on the range of environment stiffnesses the system can stably interact with. Based on experimental testing, the factor $c$ is set to 0.015 as further increasing of this factor did not result in further improvement of the system's stability. For all parameters described above, the experimental setup is stable for interaction with stiffnesses up to at least [7000-8000] N/m.

\subsection{The gSRC scheme}

This section describes the practical implementation of the controller defined in Section 3.2. Except for the parameters of the estimator, the implementation is the same as described in the former section. The tuning of the estimator has to be revised as the function $K \hat{k}_{e}^{\sigma}$ has an amplifying effect on the noise of the estimate $\hat{k}_{e}$. Figure 8 shows $\hat{k}_{e}$ and $K \hat{k}_{e}^{\sigma}$ for $\sigma=3$ and $K=\frac{1}{500^{2}}$, for the parameters selected above $\left(q_{i}=0.1\right.$ and $\left.j=12\right)$. The input for the estimator employs the same simulation data as shown in figure 5(a). From this figure, it is clear that the signal-to-noise ratio of $K \hat{k}_{e}^{\sigma}$ is worse than the signal-to-noise ratio of $\hat{k}_{e}$. This is actually obvious as the goal of the function $K \hat{k}_{e}^{\sigma}$ is to increase the relative differences in stiffness. Based on this finding, the values of the covariance matrix $Q$ should be reduced in the case that $\sigma>1$. Here, the covariance matrix has been set to:

$$
Q=\left[\begin{array}{c}
(0.03 N)^{2} \\
(0.03 N / m)^{2}
\end{array}\right]
$$

In order to maintain a sufficiently fast (and correct) response of the estimator, the delay between the two data sets has to be increased if the values of the covariance matrix $Q$ decrease

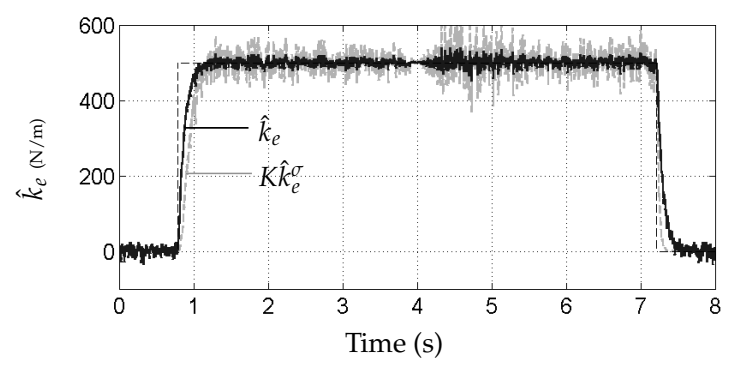

Fig. 8. The estimate $\hat{k}_{e}$ and $K \hat{k}_{e}^{\sigma}$ for the simulation data in Figure 5(a) but with white-noise added to the force measurement signal $\left(\sigma=3\right.$ and $\left.K=\frac{1}{500^{2}}\right)$. 
(see 5(c) and 5(d)). The time shift between the two data sets is set to $\left(30 \cdot T_{s}\right)$. With these values, the stiffness presented to the human operator $k_{t h}\left(=K \hat{k}_{e}^{\sigma}\right)$ behaves sufficiently smooth and accommodates sufficiently fast to changes in the environment stiffness for $\sigma \epsilon[1-3]$.

The scaling factor $K$ has to be used to maintain the absolute value of the stiffness presented to the human operator at a similar value as the real environment stiffness. Hereto, the nominal environment stiffness has to be known or estimated a priori.

\section{Experimental results: part I}

As stated in the introduction, controllers of the third concept show better robustness compared to controllers of the first concept. The experiments described in this section compare the Stiffness Reflecting Controller (SRC), a controller of the third concept to the Direct Force Feedback Controller (DFF), a controller of the first concept. A number of experiments are performed on the experimental master-slave setup described in Section 3: a comparison of the two controllers (subsection 5.1), a comparison of the two controllers when a low-pass filter with cutoff frequency of $3.2 \mathrm{~Hz}$ is present (subsection 5.2), and a comparison of the two controllers when a $100 \mathrm{~ms}$ time delay was introduced on both the control and the communication channel (subsection 5.3). Moreover, a last experiment shows the interaction with a nonlinear environment, having the material properties of typical cardiovascular tissue (subsection 5.4).

\subsection{SRC vs. DFF}

During this experiment, a linear tension spring $\left(k_{e}=1100 \mathrm{~N} / \mathrm{m}\right)$ is manipulated. Figure 9(a) and $9(\mathrm{~b})$ show the experimental data acquired during this manipulation for respectively the DFF and the SRC. For the DFF, as mentioned in Section 3.3, the stiffness felt by the operator is the series connection of the environment stiffness $\left(k_{e}\right)$ and the stiffness of the position controller $\left(K_{p}\right)$ following expression (16). Linear curve fitting shows that the stiffness presented to the operator is $\pm 850 \mathrm{~N} / \mathrm{m}$. This approximates the expected value of $\left(\frac{1}{k e}+\frac{1}{K p}\right)^{-1}$. Thus, despite good force tracking and acceptable position tracking the stiffness felt by the operator is significantly lower than the real environment stiffness. For the SRC, however, the stiffness felt by the operator is nearly the correct environment stiffness, although neither the positions nor the forces do correspond well. This example stresses the importance of displaying force position plots when analyzing stiffness transparency.

\subsection{SRC vs. DFF: a low-pass filter in the loop}

During this experiment, the same linear tension spring $\left(k_{e}=1100 \mathrm{~N} / \mathrm{m}\right)$ is manipulated, but a low-pass filter (cut-off frequency: $3.2 \mathrm{~Hz}$ ) has been added to the control channel, i.e. the position of the master is filtered before it is used as position command at the slave. In literature, the use of low-pass filters for elimination of surgical tremor is often mentioned as one of the benefits of telesurgery (Hockstein et al., 2007; Okamura, 2004). Moreover, low-pass filters can also be used to avoid excitation of the structural resonance frequencies of the slave. However, one should realize that such low-pass filters can jeopardize the implementation of haptic feedback, especially for controllers of the first and the second concept. For the DFF e.g., a low-pass filter in the loop has a negative effect on both the transparency and the stability of the overall system. Fite et al. (2001) show that the introduction of a lead filter has a positive effect on the stability of the overall system while Willaert et al. (2009b) show that velocityfeedforward to the slave also improves the stability. Both approaches decrease the total phaselag of the position controller in the loop. In contrast to this, the introduction of a low-pass filter in the control channel increases the total phase-lag and thus, has a negative effect on the 

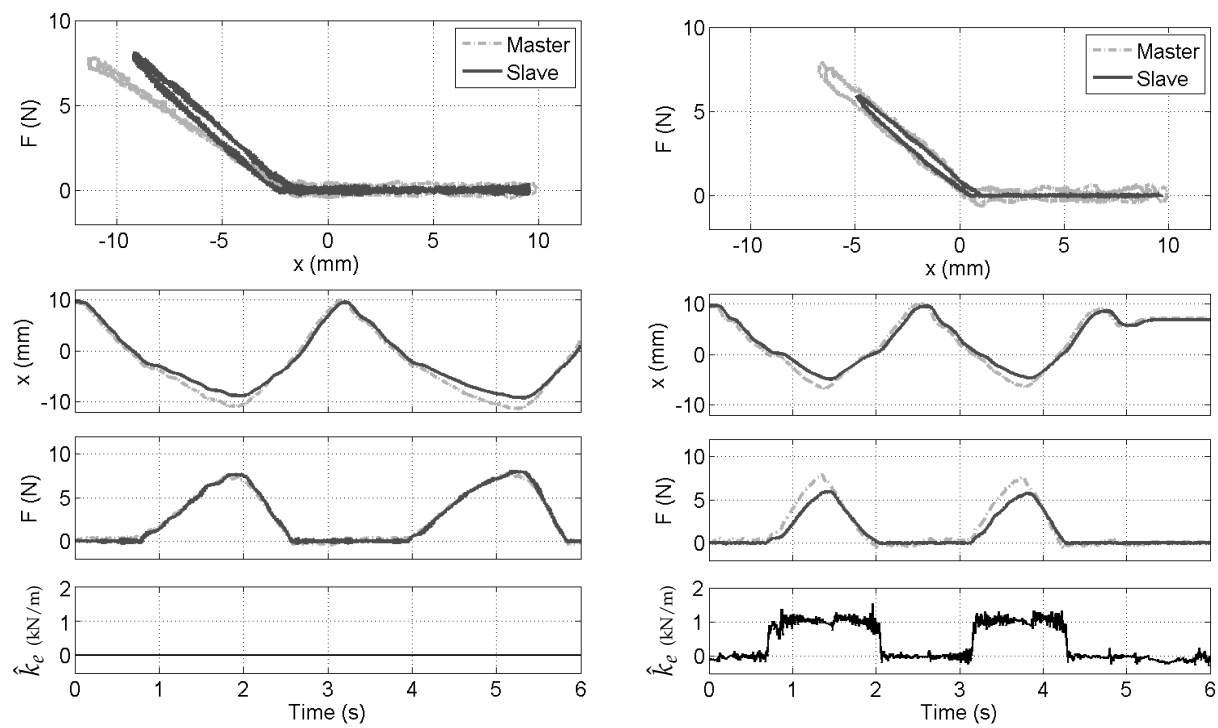

(a)

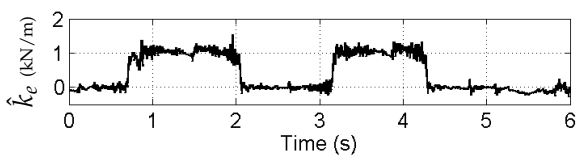

(b)

Fig. 9. Manipulation of a linear spring $\left(k_{e}=1100 \mathrm{~N} / \mathrm{m}\right)$ for (a) the DFF and (b) the SRC.

system's stability.

Figure 10(a) shows the experimental data acquired during this manipulation with a low-pass filter in the loop for the SRC. One can see that the remote environment is presented well to the human operator. Moreover, the stability is not jeopardized by the presence of the lowpass filter in the loop. With the DFF, however, the effect of the low-pass filter is clearly felt by the human operator and only for small environment stiffnesses $\left(k_{e}<200 \mathrm{~N} / \mathrm{m}\right)$, the human operator is able to keep the system stable.

\subsection{SRC vs. DFF: time-delay in the loop}

During this experiment, the same linear tension spring $\left(k_{e}=1100 \mathrm{~N} / \mathrm{m}\right)$ is manipulated, but $100 \mathrm{~ms}$ time delay has been introduced into the control and the communication channel. This means a round-trip time delay of $200 \mathrm{~ms}$. Time delay is often mentioned as an important aspect of telesurgery (C.R.Doarn et al., 2007; Lum et al., 2009; Rayman et al., 2006), although the current practice is that both the master and the slave are located in the same surgical room. As mentioned in the introduction, different controllers of the third concept have been presented in order to provide useful haptic feedback for time-delayed systems (Funda \& Paul, 1991; Hashtrudi-Zaad \& Salcudean, 1996; Ji et al., 2005; Kuan \& Young, 2003; Mitra \& Niemeyer, 2008; Tzafestas et al., 2008). Note that the estimates $\hat{k}_{e}$ and $\hat{f}_{o}$ are transferred from the slave to the master through the communication channel. Figure 10(b) shows the experimental data acquired during this manipulation with time-delay in the loop for the SRC. One can see that the human operator can feel the correct environment stiffness. As the stiffness is rendered at the master with a rest position corresponding to the real rest position, the human operator initially overshoots this rest position due to the time delay. The size of this overshoot depends on the round-trip time delay and the velocity of the master $\left(\dot{x}_{m}= \pm 21 \mathrm{~mm} / \mathrm{s}\right.$ at the first contact and $\dot{x}_{m}= \pm 30 \mathrm{~mm} / \mathrm{s}$ at the second contact). Especially in constrained environments, 

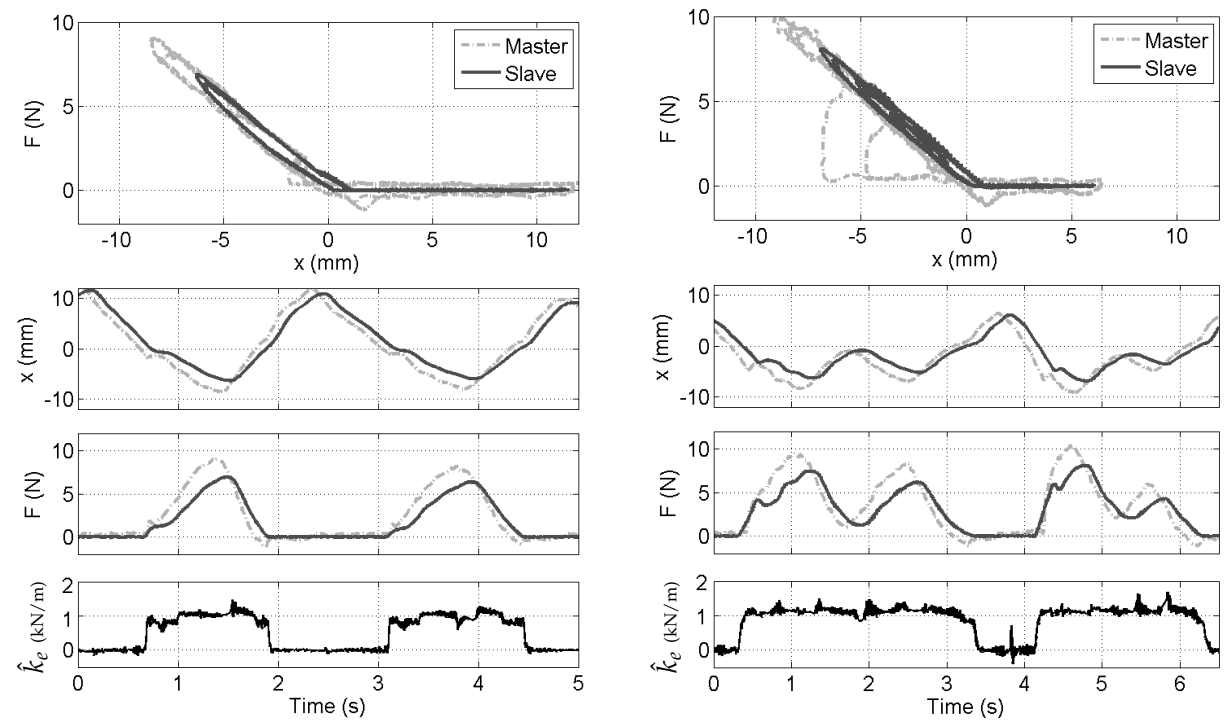

(a)

(b)

Fig. 10. Manipulation of a linear spring $\left(k_{e}=1100 \mathrm{~N} / \mathrm{m}\right)$ for the SRC with (a) a low-pass filter in the loop $(3.2 \mathrm{~Hz})$ and (b) time-delay in the loop $\left(T_{d, 1}=T_{d, 2}=100 \mathrm{~ms}\right)$.

this approach has the advantage that the human operator can directly be aware of the relative distance between different objects. With the DFF, the effect of the time-delay is similar to the effect of the low-pass filter. The time-delay is clearly felt by the human operator and only for small environment stiffnesses, the human operator is able to keep the system stable. Note that no figures are shown for the DFF with a low-pass filter or time-delay in the loop.

\subsection{SRC vs. DFF: representation of a nonlinear environment}

During this experiment, a material with nonlinear material properties is manipulated. Instead of real soft tissue as in (Willaert et al., 2008b), a Dacron cardiovascular prosthesis is used, i.e. a material with longitudinal material properties similar to cardiovascular soft tissue (GrandeAllen et al., 2001). The detail in figure 4 shows how the Dacron prosthesis is clamped as a whole and stretched in the longitudinal direction. Figure 11(a) and 11(b) show the experimental data acquired during this manipulation for respectively the DFF and the SRC. The force-position plots demonstrate that the nonlinear behaviour of the material is well reflected to the master for both controllers. But as mentioned before, the stiffness perceived with the DFF is always lower than the actual stiffness. This problem does not occur when using the SRC. This data shows that the localized linear model (11) is able to reflect a nonlinear environment reliably to the master. Note that only at the moment of motion reversal, the stiffness felt at the master is inaccurate. This due to a combination of a position tracking lag of the slave with respect to the master and an estimation lag for the abrupt change in environment stiffness as explained in Section 4.1. 

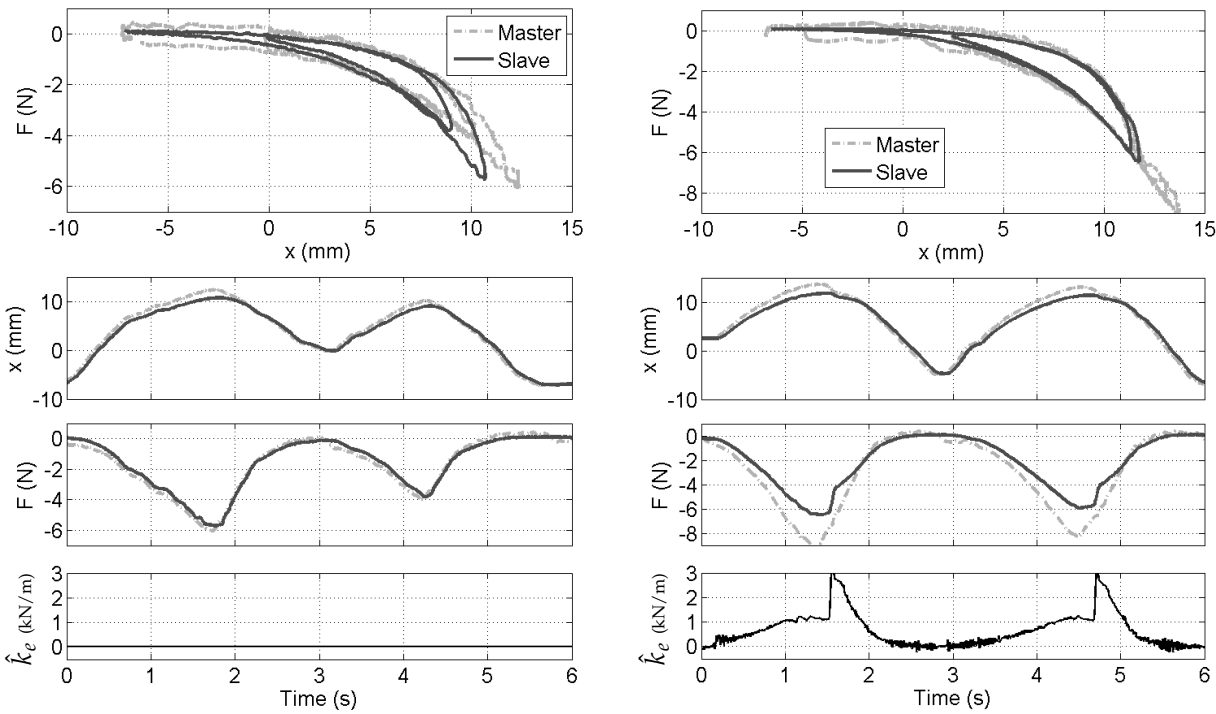

(a)

(b)

Fig. 11. Manipulation of a nonlinear material for (a) the DFF and (b) the SRC.

\section{Experimental results: part II}

The experiments, described in this section, show the potential of the generalized Stiffness Reflecting Controller (gSRC). Firstly, an interaction with a linear spring is described (subsection 6.1). Next, a psychophysical experiment is described, demonstrating the feasibility of enhanced stiffness sensitivity (subsection 6.2).

\section{1 the gSRC: stiffness shaping for interaction with a linear spring}

During this experiment, a linear tension spring $(500 \mathrm{~N} / \mathrm{m})$ is manipulated. The stiffness presented to the operator is shaped following expression (7) with $\sigma=3$ and $K=4.5 * 10^{-6}$. As a consequence, the stiffness felt by the operator should be $562 \mathrm{~N} / \mathrm{m}$. Fig. 12 shows the experimental data. Linear curve fitting on these data shows that the stiffness felt by the operator is $568 \mathrm{~N} / \mathrm{m}$, which only minimally deviates from the desired value of $562 \mathrm{~N} / \mathrm{m}$.

However, one can see a clear error during the initial contact. It takes about $0.5 \mathrm{sec}$ before the correctly shaped stiffness is felt by the operator. This behaviour can be explained by the combined effect of the estimator tuned to be less reactive (smaller process noise) and the following: when the slave makes contact with a spring, the estimated stiffness $\hat{k}_{e}$ converges from 0 to $k_{e}$, the real stiffness of the environment, during a periode of time (here in about $0.5 \mathrm{sec}$, see Fig. 12). The stiffness presented to the operator, follows expression (7) and, as mentioned in Section $4.1, K$ serves as a scaling factor to keep $k_{t h}$ at a similar value as $k_{e}$. Thus, typically $K \simeq \frac{1}{\bar{k}_{e}^{(\sigma-1)}}$, with $\bar{k}_{e}$ an a priori determined estimate of the real environment stiffness. As a result, the nonlinear mapping delays the convergence of $k_{t h}$ to the correctly shaped stiffness:

$$
k_{t h} \simeq \frac{\hat{k}_{e}(t)^{\sigma}}{\bar{k}_{e}^{(\sigma-1)}}<\hat{k}_{e}(t) \quad \text { for } \quad\left\{\begin{array}{l}
\hat{k}_{e}(t)<\bar{k}_{e} \\
\sigma>1
\end{array}\right.
$$



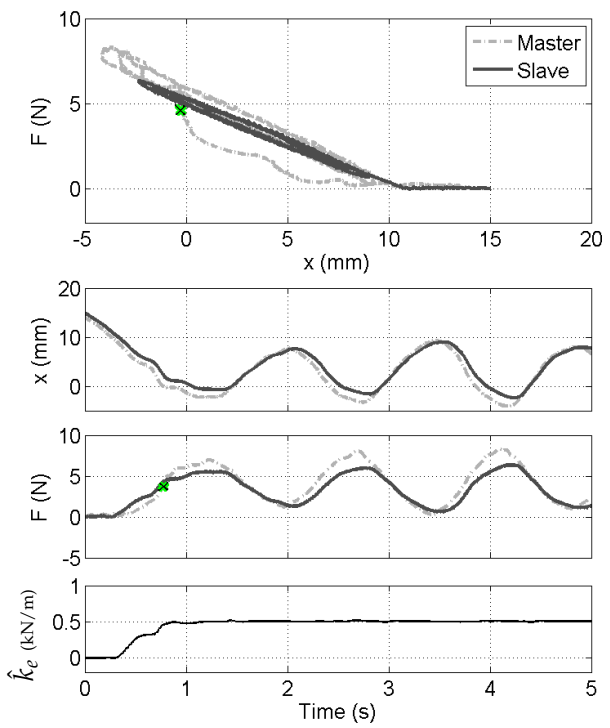

Fig. 12. Manipulation of a linear spring with shaped stiffness reflection: the force-position curve, the force vs. time, the position vs. time and the estimated environment stiffness $\hat{k}_{e}$ for the SSRC with $\sigma=3$ and $K=4.5$. The green $\operatorname{dot}(\mathrm{x})$ shows two data-points from the same time step.

\section{2 the gSRC: a psychophysical experiment}

During a psychophysical experiment six different subjects performed a stiffness differentiating task. They were asked to interact with two different springs through the master slave system, after which they had to say which spring was the stiffest. A two-alternatives forced choice procedure was employed. During the tests, the subjects could not see the slave and received no feedback from the examinator about their performance. Each subject did a total of 18 comparisons: 6 times with $\sigma=1,6$ times with $\sigma=2$ and 6 times with $\sigma=3$. These 18 test were randomized. The subjects were not informed about when and how many times each condition occurred.

The two springs had a stiffness of $182 \mathrm{~N} / \mathrm{m}$ and $197 \mathrm{~N} / \mathrm{m}$. This is a relative difference of $7.6 \%$, which lies below the practical discrimination threshold (i.e. $8 \%-12 \%$ ). For $\sigma=1, K$ is set to 1 , this is the special case of the gSRC, corresponding to the SRC. Enhanced stiffness sensitivity is offered for the cases $\sigma=2$ and $\sigma=3$. Then, $K$ is set to 0.0053 and 0.028 respectively, in order to have the perceived stiffness in the range of the real environment stiffness. Table 2 shows how each spring is presented at the master side under the three different conditions. Moreover, Table 2 shows the average percentage of correct answers for each test condition. For $\sigma=1$, the subjects were right $53 \%$ of the time. This corresponds to pure guesswork. For $\sigma=2$ and $\sigma=3$ however, i.e. with enhanced sensitivity, the average percentage of correct answers is $80 \%$ and $94 \%$ respectively. This demonstrates the ability to shape the reflected stiffness through a master-slave setup in such a way that the operator's discrimination ability is augmented. Moreover, this confirms the finding in De Gersem et al. (2005a) that the mini- 


\begin{tabular}{|c|c|c|c|}
\hline & $\sigma=1$ & $\sigma=2$ & $\sigma=3$ \\
\hline Spring 1 & $182 \mathrm{~N} / \mathrm{m}$ & $175 \mathrm{~N} / \mathrm{m}$ & $169 \mathrm{~N} / \mathrm{m}$ \\
Spring 2 & $197 \mathrm{~N} / \mathrm{m}$ & $205 \mathrm{~N} / \mathrm{m}$ & $214 \mathrm{~N} / \mathrm{m}$ \\
\hline$\delta$ & $7.6 \%$ & $14.6 \%$ & $21 \%$ \\
\hline \hline $\mathrm{P}$ & $53 \%$ & $80 \%$ & $94 \%$ \\
\hline
\end{tabular}

Table 2. Results of the Psychophysical Experiment. $\delta$ is the relative difference between the two springs felt at the master, $\mathrm{P}$ is the percentage of correct differentiation.

mal difference in stiffness that can just be discriminated is larger than $8 \%-12 \%$ of the nominal stiffness.

\section{Discussion}

The main goal of this chapter is to demonstrate the potential benefits of controllers of the third concept, i.e. controllers with model-based haptic feedback, especially for telesurgical applications. Hereto, this chapter describes the practical implementation of the Stiffness Reflecting Controller. The experiments described in Section 5 support the claim that such controllers show good robustness properties. It is shown that, for the SRC, the compliance of the position controller does not influence the stiffness felt by the operator. It is also shown that the introduction of a low-pass filter or non-negligible time-delay only minimally affects the transparency and stability for the SRC. Although not explicitly demonstrated in this chapter, controllers of the third concept can also behave more robust with respect to other hardware-related issues of surgical slave robots that traditionally restrict the applicability of bilateral controllers on such robots. Willaert et al. (2009b) show e.g. that the inertia of the slave has a large influence on the stability properties of the DFF controller and conclude that the slave inertia should be as low as possible. Since current commercial surgical robots are mostly not lightweight robots, the SRC can be a useful controller for these robots. Another, hardware aspect of current surgical robots is the restricted structural stiffness, which influences both transparency and stability (Christiansson \& van der Helm, 2007; Tavakoli \& Howe, 2009). For the DFF and a slave with flexibilities, the stiffness that the human operator feels is a series connection of the real environment stiffness, the stiffness of the position controller and the structural stiffness of the slave. In this work, the estimation of the environment stiffness is based on the force measurement at the end-effector $\left(F_{e}\right)$ and the position measurement at the motor $\left(x_{s} \approx x_{e}\right)$. As the 1 d.o.f master and slave behave as a rigid-body for frequencies below $100 \mathrm{~Hz}$, the correct environment stiffness can be estimated. For flexible multi-d.o.f. systems, however, the estimation of $k_{e}$ should be based on the force measurement at the end-effector $\left(F_{e}\right)$ and the position measurement at the end-effector $\left(x_{e} \neq x_{s}\right)$. In future research, it will be investigated how the position of the end-effector can be measured or estimated. By doing so, the SRC can be made insensitive to both the compliance of the position controller and the compliance of the slave robot itself.

Based on the detailed stability analysis of the DFF and the experiments presented here, it is clear that, compared to the DFF, the SRC will have significantly better stability properties when implemented on multi-d.o.f master-slave setups. But, a detailed analysis of the stability properties of the SRC is not straightforward due to the presence of the Extended Kalman Filter. Thanks to the introduction of a stiffness-depending damping term (see 12), the SRC implemented on the setup described in this chapter is stable for environment stiffnesses up to 
$8000 \mathrm{~N} / \mathrm{m}$. However, for real hard contacts, stability cannot be guaranteed for the SRC. Some time-domain stabilization approaches could be added to the SRC to maintain stability, also when contacting such real hard objects (Franken et al., 2009; Hannaford \& Ryu, 2001; Ryu et al., 2007; Willaert et al., 2008a).

Next, some other points of attention related to the SRC are discussed. Firstly, the master described in this chapter is of the impedance type and the desired force $f_{\text {des }}$ is sent in open loop. As a consequence the operator always feels the full dynamics (damping/friction and mass) of the master. As damping/friction can deteriorate the haptic feedback, it should be restricted. This can be done through mechanical design or by using friction compensation techniques (Tjahjowidodo et al., 2007). Here, the friction level of the master of the experimental setup is $\pm 0,5 \mathrm{~N}$. Although the friction is clearly visible on the figures, this is only hardly perceived by the operator.

Secondly, with the SRC, the interaction force at the master side $F_{h}$ is typically larger than the interaction force at the slave side $F_{e}$. This is a consequence of the position tracking error. The larger the proportional gain $K_{p}$ can be, the smaller the difference between $F_{h}$ and $F_{e}$. For an infinitely stiff position controller, the transparency will be similar for both the SRC and the DFF, in case no low-pass filter or time-delay is introduced. The fact that the interaction force $F_{h}$ is larger than the interaction force $F_{e}$ should not be too problematic since in surgery, especially during palpation, the perception of the absolute force is less important than the perception of stiffness. Moreover, a larger $F_{h}$ is in a sense safer as the environment will be subjected to lower forces than the ones applied by the operator.

\section{Conclusions}

The development of a telesurgical system providing reliable force feedback forms a real challenge. First, such a development requires the design of an appropriate master and slave, applicable in the surgical theatre, and the development of robust force measurement systems. Second, a reliable controller, providing a transparent and guaranteed stable system is required. The latter is addressed in this chapter.

Based on the idea that the perception of stiffness of tissues plays an essential role in the decision making process during surgery, this chapter explains the concepts of stiffness transparency and enhanced stiffness sensitivity. A practical implementation of a model-based haptic feedback approach is presented and discussed, referred to as the (generalized) Stiffness Reflecting Controller (SRC). The SRC employs a spring with variable stiffness and rest position as model for the environment. It was shown, that such a model-based haptic feedback has good robustness properties with respect to time-delay. However, the robustness of this approach is not restricted to time-delay. The experiments presented in this chapter demonstrate that the SRC is also very suitable to realize good stiffness transparency for both linear and nonlinear environments, even when the slave shows limited responsiveness in terms of position tracking. This limited responsiveness can originate from either the hardware of the slave (e.g. a large inertia), the control of the slave (e.g. restricted gains) or a low-pass filter in the control channel (e.g. to avoid the transmission of surgical tremor). A topic to address in the future, is how model-based haptic feedback can be employed to increase the robustness with respect to flexibilities of current surgical slave robots. 
Next to the increased robustness, the approach of model-based haptic feedback offers the possibility to shape the environment stiffness before it is reflected to the human operator. Human stiffness perception is limited by both absolute and differential thresholds. Enhanced stiffness sensitivity allows to overcome the differential threshold through master-slave control in order to increase the stiffness discrimination ability of the human operator. This chapter describes a practical implementation of the generalized version of the Stiffness Reflecting Controller realizing enhanced stiffness transparency. The psychophysical experiments with this controller demonstrate the feasibility of enhanced stiffness sensitivity for linear environments. Further investigations are necessary to determine how to enhance sensitivity when contacting objects with nonlinear stiffness properties.

\section{Acknowledgement}

This work was supported by a PhD grant from the Institute for the Promotion of Innovation through Science and Technology in Flanders (I.W.T.-Vlaanderen), one I.W.T project (IWT/OZM/080086) and by the K.U.Leuven BOF-IDO/05/008 project as well as by an FP7People Marie Curie Reintegration Grant, PIRG03-2008-231045.

\section{References}

Bankman, I., Gruhen, K., Halperin, H., Popel, A., Guerci, A. \& Tsitlik, J. (1990). Identification of dynamic mechanical properties of the human chest during manual cardiopulmonary resuscitation, IEEE Transactions on Biomedical Engineering 37(2): 211-217.

Bethea, B., Okamura, A., Kitagawa, M., Fitton, T., Cattaneo, S., Gott, V., Baumgartner, W. \& Yuh, D. (2004). Application to haptic feedback to robotic surgery, J Laparoendosc Adv Surg Tech A. 14: 191-195.

Cavusoglu, M., Sherman, A. \& Tendick, F. (2002). Bilateral controller design for telemanipulation in soft environments, IEEE Transactions on Robotics and Automation 18(4): 641-647.

Christiansson, G. \& van der Helm, F. (2007). The low-stiffness teleoperator slave - a trade-off between stability and performance, Int. Journal of Robotics Research 26(3): 287-299.

Colgate, J. E. (1993). Robust impedance shaping telemanipulation, IEEE Transactions on robotics and automation 9(4): 374-384.

Corcione, F., Esposito, C., Cuccurullo, D., Settembre, A., Miranda, N., Amato, F., Pirozzi, F. \& Caiazzo, P. (2005). Advantages and limits of robot-assisted laparoscopic surgery, Surgical Endoscopy 19: 117-119.

C.R.Doarn, K.Hufford, Rosen, T. L. J. \& B.Hannaford (2007). Telesurgery and robotics: A roundtable discussion., Telemed J E Health 13(4): 369-380.

De Gersem, G., Van Brussel, H. \& Tendick, F. (2005a). Reliable and enhanced stiffness perception in soft-tissue telemanipulation, The international Journal of Robotics Research 24(10): 805-822.

De Gersem, G., Van Brussel, H. \& Vander Sloten, J. (2005b). Enhanced haptic sensitivity for soft tissues using teleoperation with shaped impedance reflection, Proceedings of the World Haptics Conference, Pisa, Italy.

De Gersem, G. (2005). Kinaesthetic feedback and enhanced sensitivity in robotic endoscopic telesurgery, PhD thesis, Katholieke Universiteit Leuven.

De, S., Rosen, J., Dagan, A., Hannaford, B., Swanson, P. \& Sinanan, M. (2007). Assessment of tissue damage due to mechanical stresses, The Int. Journal of Robotics Research 26(1112): 1159-1171. 
De Schutter, J., Greeter, J. D., Lefebvre, T. \& Bruyninckx, H. (1999). Kalman filters: a tutorial, Journal E 40(40): 52-59.

Deml, B., Ortmaier, T. \& Seibold, U. (2005). The touch and feel in minimally invasive surgery, Proceedings of International Workshop on Haptic Audio Visual Environments and their Applications, Ontario, Canada, pp. 33-38.

Famaey, N., Verbeken, E., Vinckier, S., Willaert, B., Herijgers, P. \& Vander Sloten, J. (2009). In vivo soft tissue damage assessment for applications in surgery. Submitted to Medical Engineering and Physics.

Fite, K., Speich, J. \& Goldfarb, M. (2001). Transparency and stability robustness in twochannel bilateral telemanipulation, Journal of Dynamic Systems, Measurement, and Control 123: 400-407.

Franken, M., Stramigioli, S., Reilink, R., Secchi, C. \& Macchelli, A. (2009). Bridging the gap between passivity and transparency, Proceedings of Robotics: Science and Systems, Seattle, USA.

Funda, J. \& Paul, R. (1991). Efficient control of a robotic system for time-delayed environments, Fifth International Conference on Advanced Robotics (ICAR), Pisa, Italy, pp. 219-224.

Grande-Allen, K. J., Cochran, R. P., Reinhall, P. G. \& Kunzelman, K. S. (2001). Finite-element analysis of aortic valve-sparing: influence of graft shape and stiffness., IEEE Trans Biomed Eng 48(6): 647-659.

Handlykken, M. \& Turner, T. (1980). Control system analysis and synthesis for a six degree-offreedom universal force-reflecting hand controller, Proceedings of the IEEE Conference on Decision and Control, pp. 1197-1205.

Hannaford, B. (1989). A design framework for teleoperators with kinesthetic feedback, IEEE Transactions on Robotics and Automation 5(4): 426-434.

Hannaford, B. \& Ryu, J. (2001). Time domain passivity control of haptic interfaces, Proceedings of the IEEE International Conference on Robotics and Automation, Seoul, Korea, pp. 18631869.

Hashtrudi-Zaad, K. \& Salcudean, S. (1996). Adaptive transparent impedance reflecting teleoperation, Proceedings of The IEEE International Conference on Robotics and Automation, Minneapolis, Minnesota, pp. 1369-1374.

Hockstein, N., Gourin, C., Faust, R. \& Terris, D. (2007). A history of robots: from science fiction to surgical robotics, Journal of Robotic Surgery 1: 113-118.

Ji, H., Song, A., Liu, W. \& Li, J. (2005). Dynamic vr modeling for force-reflecting teleoperation with time delay, Proceedings of the IEEE International Conference on Information Acquisition, Hong Kong, China, pp. 32-36.

Kalman, R. (1960). A new approach to linear filtering and prediciton problems, Journal of Basic Engineering pp. 35-45.

Kuan, C.-P. \& Young, K.-Y. (2003). Challenges in vr-based robot teleoperation, Proceedings of the IEEE International Conference on Robotics and Automation, Taipei, Taiwan, pp. 43924397.

Lawrence, D. (1993). Stability and transparency in bilateral teleoperation, IEEE transactions on robotics and automation 9(5): 624-637.

Love, L. \& Book, W. J. (2004). Force reflecting teleoperation with adaptive impedance control, IEEE Transactions on Systems, Man and Cybernetics - Part B: Cybernetics 34(1): 159-165.

Lum, M. J., Rosen, J., Lendvay, T. S., Sinanan, M. N. \& Hannaford, B. (2009). Effect of time delay on telesurgical performance, Proceedings of the International Conference on Robotics and Automation, Kobe, Japan. 
Mahvash, M. \& Okamura, A. (2007). Friction compensation for enhancing transparency of a teleoperator with compliant transmission, IEEE Transactions on Robotics 23: 12401246.

Malysz, P. \& Siroupour, S. (2007). Stable non-linear force/position mapping for enhanced telemanipulation of soft environments, Proceedings of the International Conference on Robotics and Automation, Roma, Italy, pp. 4307-4312.

Marescaux, J., Leroy, J., Gagner, M., Rubino, F., Mutter, D., Vix, M., Butner, S. E. \& Smith, M. K. (2001). Transatlantic robot-assisted telesurgery, Nature 413: 379-380.

Misra, S. \& Okamura, A. (2006). Environment parameter estimation during bilateral telemanipulation, Proceedings of Symposium on Haptic Interfaces for Virtual Environment and Teleoperator Systems, Virginia, USA, pp. 301-307.

Mitra, P. \& Niemeyer, G. (2008). Model-mediated telemanipulation, Int. Journal of Robotics Research 27(2): 253-262.

Nguan, C., Girvan, A. \& Luke, P. (2008). Robotic surgery versus laparoscopy; a comparison between two robotic systems and laparoscopy, Journal of Robotic Surgery 1: 263-268.

Okamura, A. (2004). Methods for haptic feedback in teleoperated robot-assisted surgery, Industrial Robot: An international Journal 31(6): 499-508.

Ota, D. (1995). Laparoscopic colectomy for cancer: a favorable opinion, Ann. Surgical Oncology 2: 3-5.

Peirs, J., Clijnen, J., Reynaerts, D., Brussel, H. V., Herijgers, P., Corteville, B. \& Boone, S. (2004). A micro-optical force sensor for force feedback during minimally invasive robotic surgery., Sensor and Actuators A 115: 447-455.

Preusche, C., Ortmaier, T. \& Hirzinger, G. (2002). Teleoperation concepts in minimal invasive surgery, Control Engineering Practice 10(11): 1245-1250.

Rayman, R., Croome, K., Galbraith, N., McClure, R., Morady, R., Peterson, S., Smith, S., Subotic, V., Wynsberghe, A. V. \& Primak, S. (2006). Long-distance robotic telesurgery: a feasibility study for care in remote environments, Int J Med Robotics Comput Assist Surg 2: 216-224.

Rosen, J., Jeffrey, D., De, S., Sinanan, M. \& Hannaford, B. (2008). Biomechanical properties of abdominal organs in vivo and postmortem under compression loads, Journal of Biomechanical Engineering 130(2): 1-17.

Ryu, D., Song, J.-B., Choi, J., Kang, S. \& Kim, M. (2007). Frequency domain stability observer and active damping control for stable haptic interaction, Proceedings of the IEEE International Conference on Robotics and Automation, Roma, Italy, pp. 105-110.

Scott, H. \& Darzi, A. (1997). Tactile feedback in laparoscopic colonic surgery, The Britisch Journal of Surgery 84: 1005.

Seibold, U., Kübler, B. \& Hirzinger, G. (2005). Prototype of instrument for minimally invasive surgery with 6-axis force sensing capability, Proceedings of the IEEE International Conference on Robotics and Automation, Barcelona, Spain, pp. 496-501.

Son, H. I. \& Lee, D. Y. (2008). Enhancement of kinesthetic perception for microsurgical teleoperation using impedance-shaping, Proceedings of the 30th Annual Int. IEEE EMBS Conference, Vancouver, B.C., Canada, pp. 1939-1942.

Tavakoli, M. \& Howe, R. (2009). Haptic effect of surgical teleoperator flexibility, Int. Journal of Robotics Research.

Tavakoli, M., Patel, R. \& Moallem, M. (2006). A haptic interface for computer-integrated endoscopic surgery and training, Virtual Reality 9: 160-176. 
Tholey, G., Desai, J. \& Castellanos, A. (2005). Force feedback plays a significant role in minimally invasive surgery, Annals of surgery 241(1): 102-109.

Tjahjowidodo, T., Al-Bender, F., Van Brussel, H. \& Symens, W. (2007). Friction characterization and compensation in electro-mechanical systems, Journal of Sound and Vibration 308: 632-646.

Tzafestas, C., Velanas, S. \& Fakiridis, G. (2008). Adaptive impedance control in haptic teleoperation to improve transparency under time-delay, Proceedings of the IEEE International Conference on Robotics and Automation, Pasadena, CA, USA, pp. 212-219.

Wagner, C. R., Stylopoulos, N. \& Howe, R. D. (2002). The role of force feedback in surgery: Analysis of blunt dissection, The 10th Symp. on Haptic interfaces for virtual environment and teleoperator systems, Orlando.

Walraevens, J., Willaert, B., De Win, G., Ranftl, A., De Schutter, J. \& Vander Sloten, J. (2008). Correlation between compression, tensile and tearing tests on healthy and calcified aortic tissues, Medical Engineering \& Physics 30: 1098-1104.

Weber, E. H. (1834). De pulsu, resorptione, auditu et tactu, Annotationes Anatomicae Et Physiologicae pp. 19-135.

Willaert, B., Vander Poorten, E., Reynaerts, D. \& Van Brussel, H. (2008a). A pragmatic method for stable stiffness reflection in telesurgery, Haptics: Perception, Devices and Scenarios (6th Int. Conf, Eurohaptics), Madrid, Spain, pp. 73-82.

Willaert, B., Vander Poorten, E., Reynaerts, D. \& Van Brussel, H. (2008b). Reliable stiffness reflection for telesurgery, ICRA 2008 Workshop on new vistas and challenges in Telerobotics, Pasadena, CA, USA.

Willaert, B., Reynaerts, D., Van Brussel, H. \& Vander Poorten, E. (2009a). Extracorporeal force measurement system for robot-assisted mis with 3d force feedback, ICRA 2009 Workshop on Advanced Sensing and Sensor Integration in Medical Robotics, Kobe, Japan.

Willaert, B., Corteville, B., Reynaerts, D., Van Brussel, H. \& Vander Poorten, E. (2009b). Bounded environment passivity of the classical position-force controler, Proceedings of the IEEE International Conference on Intelligent Robots and Systems, St. Louis, MO, USA, p. 4622 - 4628

Yokokohji, Y. \& Yoshikawa, T. (1994). Bilateral control of master-slave manipulators for ideal kinesthetic coupling, IEEE transactions on robotics and automation 10(5): 605-620.

Zemiti, N., Ortmaier, T., Vitrani, M. \& Morel, G. (2006). A Force Controlled Laparoscopic Surgical Robot without Distal Force Sensing, Springer Berlin / Heidelberg, chapter V. Medical Robotics in Experimental Robotics IX, pp. 153-164. 


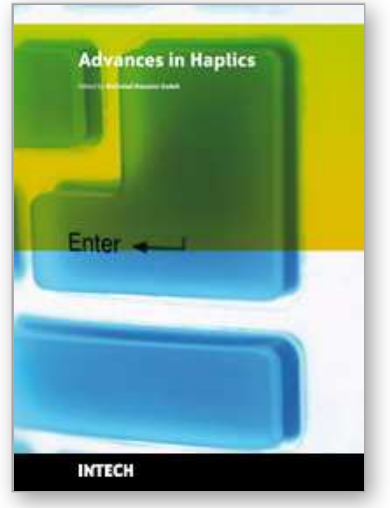

\author{
Advances in Haptics \\ Edited by Mehrdad Hosseini Zadeh
}

ISBN 978-953-307-093-3

Hard cover, 722 pages

Publisher InTech

Published online 01, April, 2010

Published in print edition April, 2010

Haptic interfaces are divided into two main categories: force feedback and tactile. Force feedback interfaces are used to explore and modify remote/virtual objects in three physical dimensions in applications including computer-aided design, computer-assisted surgery, and computer-aided assembly. Tactile interfaces deal with surface properties such as roughness, smoothness, and temperature. Haptic research is intrinsically multidisciplinary, incorporating computer science/engineering, control, robotics, psychophysics, and human motor control. By extending the scope of research in haptics, advances can be achieved in existing applications such as computer-aided design (CAD), tele-surgery, rehabilitation, scientific visualization, robot-assisted surgery, authentication, and graphical user interfaces (GUI), to name a few. Advances in Haptics presents a number of recent contributions to the field of haptics. Authors from around the world present the results of their research on various issues in the field of haptics.

\title{
How to reference
}

In order to correctly reference this scholarly work, feel free to copy and paste the following:

Bert Willaert, Pauwel Goethals, Dominiek Reynaerts, Hendrik Van Brussel and Emmanuel B. Vander Poorten (2010). Transparent and Shaped Stiffness Reflection for Telesurgery, Advances in Haptics, Mehrdad Hosseini Zadeh (Ed.), ISBN: 978-953-307-093-3, InTech, Available from: http://www.intechopen.com/books/advancesin-haptics/transparent-and-shaped-stiffness-reflection-for-telesurgery

\section{INTECH}

open science | open minds

\section{InTech Europe}

University Campus STeP Ri

Slavka Krautzeka 83/A

51000 Rijeka, Croatia

Phone: +385 (51) 770447

Fax: +385 (51) 686166

www.intechopen.com

\section{InTech China}

Unit 405, Office Block, Hotel Equatorial Shanghai

No.65, Yan An Road (West), Shanghai, 200040, China

中国上海市延安西路65号上海国际贵都大饭店办公楼405单元

Phone: +86-21-62489820

Fax: $+86-21-62489821$ 
(C) 2010 The Author(s). Licensee IntechOpen. This chapter is distributed under the terms of the Creative Commons Attribution-NonCommercialShareAlike-3.0 License, which permits use, distribution and reproduction for non-commercial purposes, provided the original is properly cited and derivative works building on this content are distributed under the same license. 